\title{
Evidências de validade da Escala de Enriquecimento Trabalho-Família em amostras brasileiras
}

\author{
Larissa Maria David Gabardo-Martins' \\ Maria Cristina Ferreira \\ Felipe Valentini \\ Universidade Salgado de Oliveira, Niterói - RJ - Brasil
}

\begin{abstract}
Resumo: $O$ enriquecimento trabalho-família diz respeito aos modos pelos quais as experiências provenientes de um domínio (trabalho ou família) interferem positivamente no outro. $\mathrm{O}$ objetivo da pesquisa é adaptar e buscar evidências de validade da Escala de Enriquecimento Trabalho-Família no contexto brasileiro. Participaram do estudo 416 trabalhadores de ambos os sexos, com idades variando de 20 a 66 anos, que responderam à versão inicial da escala, contendo 18 itens. As análises fatoriais confirmatórias evidenciaram que a versão final da escala compôs-se de dez itens, distribuídos igualmente em dois fatores, com índices adequados de consistência interna. $\mathrm{O}$ enriquecimento apresentou correlações fortes e positivas com a satisfação com a vida e com o trabalho, e moderadas e negativas com o conflito trabalho-família. Concluiu-se que a escala é um instrumento com evidências de validade adequadas, o que possibilita seu uso em situações futuras de pesquisa da interface trabalho-família.
\end{abstract}

Palavras-chave: psicologia positiva; interface trabalho-família; conflito trabalho-família; enriquecimento trabalho-família; adaptação de escala.

\section{EVIDENCES OF VALIDITY OF THE SCALE OF WORK-FAMILY ENRICHMENT IN BRAZILIAN SAMPLES}

\begin{abstract}
The work-family enrichment concerns the ways in which the experiences from one domain (work or family) interfere positively in another. The objective of the research is to adapt and get validity evidences of the scale of Work-Family Enrichment in the Brazilian context. Participated in this study 416 workers of both sexes, with ages ranging from 20 to 66 years, who responded to the initial version of the scale, containing 18 items. Confirmatory factorial analyses showed that the final version of the scale composed of ten items, distributed equally on two factors, with appropriate indexes of internal consistency. The enrichment has also presented strong and positive correlations with satisfaction with life and with the work, as well as moderate and negative correlations with the work-family conflict. The conclusion was that the scale is an instrument with adequate validity evidences, allowing its use in future situations of research of the work-family interface.
\end{abstract}

Keywords: positive psychology; work-family interface; work-family conflict; work-family enrichment; adaptation of scale.

1 Endereço para correspondência: Larissa Maria David Gabardo-Martins, Avenida Oito de Maio, 628, Centro. Silva Jardim - RJ - Brasil. Cep: 28820-000. E-mail: laragabardo@yahoo.com.br. 
EVIDENCIAS DE VALIDAD DE LA ESCALA DE ENRIQUECIMIENTO TRABAJO-FAMILIA EN MUESTRAS BRASILEÑAS

\begin{abstract}
Resumen: El enriquecimiento trabajo-familia consiste en los modos en que las experiencias provenientes de un dominio (trabajo o familia) interfieren positivamente en el otro. El objetivo de la investigación es adaptar y buscar evidencias de validad de la Escala de Enriquecimiento Trabajo-Familia en el contexto brasileño. Participaron en este estudio 416 trabajadores de ambos sexos, con edades variando de 20 a 66 años, que respondieron a la versión inicial de la escala, conteniendo 18 ítems. Los análisis factoriales confirmatorios evidenciaron que la versión final de la escala tiene diez ítems, distribuidos igualmente en dos factores, con índices adecuados de consistencia interna. El enriquecimiento presentó correlaciones fuertes y positivas con la satisfacción con la vida e el trabajo, y correlaciones moderadas y negativas con el conflicto trabajo-familia. Se concluye que la escala es un instrumento con evidencias de validez adecuada, pudiendo ser adoptada en situaciones futuras de investigación de la interface trabajo-familia.
\end{abstract}

Palabras clave: psicología positiva, interface trabajo-familia, conflicto trabajo-familia, enriquecimiento trabajo-familia, adaptación de escala.

As pesquisas sobre as inter-relações de trabalho e família dedicaram-se por muito tempo a estudar principalmente os conflitos existentes entre esses dois domínios, ignorando, assim, a ideia de que eles poderiam se beneficiar mutuamente (Greenhaus \& Parasuraman, 1999). Mais recentemente, porém, os estudiosos dessa área passaram a reconhecer que a combinação das funções do trabalho e da família costuma gerar efeitos positivos para o indivíduo (Greenhaus \& Powell, 2006).

Nesse contexto, fenômenos como a facilitação trabalho-família (Grzywacz, 2002), o spillover positivo trabalho-família (Poelmans, Stepanova, \& Masuda, 2008) e o enriquecimento trabalho-família (Greenhaus \& Powell, 2006) passaram a despertar o interesse dos investigadores. Esse último construto diz respeito aos modos pelos quais as experiências provenientes de um domínio interferem positivamente no outro, isto é, quando os recursos ganhos em um domínio promovem melhor desempenho no outro. Assim, por exemplo, o trabalho pode beneficiar a família melhorando a qualidade de vida pessoal, enquanto a família pode beneficiar o trabalho, reduzindo o estresse e melhorando a qualidade de vida no trabalho (Greenhaus \& Powell, 2006).

Um dos modelos teóricos frequentemente adotados para a explicação do enriquecimento trabalho-família é o de Greenhaus e Powell (2006). De acordo com esses autores, os recursos (habilidades e perspectivas, recursos psicológicos e físicos, recursos sociocapitais, flexibilidade e recursos materiais) gerados em um domínio A (família, por exemplo) aumentam a qualidade de vida no domínio B (trabalho, por exemplo).

$O$ domínio em que o enriquecimento tem origem (trabalho, por exemplo) é o que mais se beneficia com tal enriquecimento (leva à satisfação no trabalho, por exemplo), em razão de que os indivíduos tendem a atribuir as experiências positivas que vivenciam aos recursos gerados no domínio de origem (Carlson, Kacmar, Wayne, \& Grzywacz, 2006; Greenhaus \& Powell, 2006). Nesse sentido, o enriquecimento do trabalho para a família propicia que recursos gerados no trabalho sejam transferidos para a família, o que leva o indivíduo a obter maior satisfação com o trabalho. Por outro lado, o enri- 
quecimento da família para o trabalho leva à maior satisfação com a família. De modo consistente com tais asserções, tem-se verificado que, no que diz respeito à satisfação com o trabalho e a família, o enriquecimento do trabalho para a família impacta principalmente a satisfação no trabalho, enquanto o enriquecimento da família para o trabalho exerce maior impacto na satisfação com a família (Hennessy, 2007; McNall, Nicklin, \& Masuda, 2010).

Tomando por base a teoria de Greenhaus e Powell (2006), Carlson et al. (2006) desenvolveram, nos Estados Unidos, o primeiro instrumento destinado à avaliação do enriquecimento trabalho-família. Ele se compõe de 18 itens, divididos em duas subescalas (Enriquecimento do Trabalho para a Família e Enriquecimento da Família para o Trabalho), com cada uma delas englobando três dimensões. A subescala de Enriquecimento do Trabalho para a Família apresenta as seguintes dimensões: desenvolvimento trabalho-família (a aquisição ou o aperfeiçoamento de habilidades, conhecimentos, comportamentos e/ou modos de ver as situações no trabalho ajudam o indivíduo a ser um membro melhor da família, $\alpha=0,73, \mathrm{~N}=243$ ); afeto trabalho-família (o estado emocional ou as atitudes positivas decorrentes do trabalho ajudam o indivíduo a ser um membro melhor da família, $\alpha=0,91, \mathrm{~N}=243$ ) e capital trabalho-família (os recursos psicossociais, como a sensação de segurança, de confiança e de realização adquiridos no trabalho ajudam o indivíduo a ser um membro melhor da família, $\alpha=0,90, \mathrm{~N}=243$ ).

A subescala de Enriquecimento da Família para o Trabalho, por sua vez, é composta pelas seguintes dimensões: desenvolvimento família-trabalho (a aquisição ou o aperfeiçoamento de habilidades, conhecimentos, comportamentos e/ou pontos de vista na família ajudam o indivíduo a ser um trabalhador melhor, $\alpha=0,87, \mathrm{~N}=243$ ); afeto família-trabalho (o estado emocional ou atitudes positivas provenientes da família ajudam o indivíduo a ser um trabalhador melhor, $\alpha=0,84, \mathrm{~N}=243$ ) e eficiência família-trabalho (o sentimento de foco ou de urgência obtido na família ajuda o indivíduo a ser um trabalhador melhor, $\alpha=0,82, \mathrm{~N}=243$ ) (Carlson et al., 2006).

A Escala de Carlson et al. (2006) foi adaptada por Lim, Choi e Song (2012), para a Coreia do Sul, e Nicklin e McNall (2013) reuniram evidências adicionais sobre a validade da escala em trabalhadores norte-americanos, com ambos os estudos reproduzindo a estrutura original de duas subescalas, cada uma contendo três dimensões. No entanto, a pesquisa de Hennessy (2007), com mulheres norte-americanas, confirmou tão somente a estrutura de duas subescalas (enriquecimento do trabalho para a família e enriquecimento da família para o trabalho). O referido estudo evidenciou, ainda, a necessidade de se eliminarem quatro itens da escala de enriquecimento trabalho-família (itens $2,6,7,8$ ) e quatro itens da escala de enriquecimento família-trabalho (itens 11, 14, 16, 17), ficando ambas as escalas com cinco itens cada, as quais apresentaram, respectivamente, índices de consistência interna iguais a 0,91 e 0,79.

A maioria das investigações com a escala de Carlson et al. (2006) tem sido realizada, no entanto, em amostras norte-americanas ou asiáticas. Justificam-se, dessa maneira, estudos que possam trazer evidências adicionais acerca das características psicométri- 
cas do instrumento em amostras ainda não contempladas em pesquisas anteriores, como é o caso, por exemplo, do contexto brasileiro. Ademais, a validação da referida escala em amostras brasileiras poderá contribuir para a realização de estudos futuros sobre as implicações do enriquecimento trabalho-família para o bem-estar e a qualidade de vida dos indivíduos em ambiente laboral e familiar.

Considerando-se que o domínio no qual o enriquecimento tem origem é o que mais se beneficia com tal enriquecimento (Carlson et al., 2006), hipotetizou-se (H1) a existência de uma correlação alta e positiva entre a subescala de Enriquecimento do Trabalho para a Família e a Satisfação com o Trabalho, que diz respeito às crenças e pensamentos positivos acerca do próprio trabalho (Weiss, 2002). Adotando-se o mesmo raciocínio, foi formulada a hipótese $(\mathrm{H} 2)$ de que haveria uma correlação alta e positiva entre o Enriquecimento da Família para o Trabalho e a Satisfação com a Vida, que representa um processo de julgamento e avaliação geral da própria vida, que depende da comparação entre as circunstâncias da vida do indivíduo a um padrão por ele previamente definido (Emmons, 1986).

O conflito entre trabalho e família ocorre quando as demandas do trabalho interferem nas atividades que deveriam ser realizadas no contexto familiar ou vice-versa (Aguiar \& Bastos, 2013). Tal construto consiste, portanto, em uma consequência do processo de gerenciamento do domínio do trabalho e da família, que produz resultados diferentes dos obtidos no caso do enriquecimento trabalho-família (Hennessy, 2007). Seria assim de esperar que tais construtos se apresentassem pouco ou nada correlacionados, em razão de se constituírem em fenômenos distintos, o que de fato ocorreu na pesquisa de Hennessy (2007), que obteve uma correlação baixa e negativa $(r=-0,21)$ entre o conflito e o enriquecimento trabalho-família considerados como construtos compostos. Fundamentando-se em tais considerações, formularam-se as hipóteses de que a subescala de Enriquecimento do Trabalho para a Família apresentaria uma correlação baixa com a subescala de Interferência do Trabalho na Família (H3), e que a subescala de Enriquecimento da Família para o Trabalho apresentaria uma correlação baixa com a subescala de Interferência da Família no Trabalho (H4).

Fundamentando-se, portanto, nessas considerações, o presente trabalho teve como objetivo geral obter evidências iniciais de validade de estrutura interna, convergente e divergente da Escala de Enriquecimento Trabalho-Família em amostras brasileiras, bem como indicadores de sua consistência interna.

\section{Método}

\section{Participantes}

A amostra final ficou composta por 416 trabalhadores brasileiros, de ambos os sexos e profissões variadas, provenientes, em sua grande parte $(93,5 \%)$, do estado do Rio de Janeiro. Do total de respondentes, a maioria era de mulheres (67,8\%). Quanto ao estado civil, a maior parte era casada $(85,1 \%)$ e tinha pelo menos um filho $(71,9 \%)$. 
No que diz respeito à escolaridade, 59,6\% indicaram nível superior completo e $18,8 \%$, ensino médio completo. $A$ idade da amostra variou de 20 a 66 anos $(M=37,24 ; D P=$ $10,79)$ e o tempo de serviço, de 1 a 41 anos $(M=10,43$; $D P=10,01)$. Como critério de inclusão na amostra, a pessoa deveria ser casada e/ou ter pelo menos um filho, conforme critério também utilizado por Carlson et al. (2006).

\section{Instrumentos}

O enriquecimento trabalho-família foi avaliado por meio da escala desenvolvida por Carlson et al. (2006), que se compõe de 18 itens, a serem respondidos por meio de escalas Likert de cinco pontos, variando de "discordo fortemente" (1) a "concordo fortemente" (5). Exemplo de item da direção enriquecimento do trabalho para a família: meu envolvimento com meu trabalho me deixa alegre e isso me ajuda a ser um membro melhor da família. Exemplo de item da direção da família para o trabalho: Meu envolvimento com minha família me ajuda a ampliar meu conhecimento sobre coisas novas e isso me ajuda a ser um trabalhador melhor. Para a tradução da Escala, foi adotado o procedimento de tradução e retradução (back-translation), que consiste em uma tradução dos itens para o português, seguida da tradução dessa versão novamente para o inglês e da comparação dessas duas versões, a fim de verificar se houve uma equivalência conceitual (Borsa, Damásio, \& Bandeira, 2012).

O conflito trabalho-família foi avaliado pela Escala de Conflito Trabalho-Família de Netemeyer, Boles e McMurrian (1996), adaptada para o contexto brasileiro por Aguiar e Bastos (2013). A escala tem como objetivo identificar como o trabalho interfere na família e vice-versa. A medida é composta de dez itens, divididos em dois fatores: Interferência do Trabalho na Família e Interferência da Família no Trabalho, a serem respondidos em escalas tipo Likert de 6 pontos, variando de "discordo totalmente" (1) a "concordo totalmente" (6). Exemplo de item da dimensão interferência do trabalho na família: As demandas do meu trabalho interferem na minha vida familiar. Exemplo de item da dimensão interferência do trabalho na família: as demandas da minha família interferem nas minhas atividades de trabalho. Na adaptação da escala realizada por Aguiar e Bastos (2013), a estrutura de dois fatores se manteve e os índices de precisão (Alfa de Cronbach) foram iguais a 0,91 e 0,85, respectivamente. Na presente pesquisa, os índices de precisão (Alfa de Cronbach) foram iguais a 0,92 e 0,87, respectivamente.

$\mathrm{Na}$ avaliação da satisfação com a vida adotou-se a Escala de Satisfação com a Vida, de Diener, Emmons, Larsen e Griffin (1985), adaptada para amostras brasileiras por Gouveia Chaves, Oliveira, Dias, Gouveia e Andrade (2003). Tal escala busca mensurar a satisfação que o indivíduo tem em relação à sua vida em geral. $O$ instrumento é unifatorial e composto de cinco itens, a serem respondidos por meio de uma escala tipo Likert de sete pontos, variando de "discordo totalmente" (1) a "concordo totalmente" (7). Exemplo de item: as condições da minha vida são excelentes. $\mathrm{Na}$ adaptação para o contexto brasileiro (Gouveia et al., 2003), o índice de consistência interna, avaliado pelo Alfa de Cronbach, foi de 0,72. Na presente pesquisa, o Alfa foi igual a 0,91. 
A satisfação no trabalho foi mensurada por meio da Escala de Satisfação Geral no Trabalho, de Silva e Ferreira (2009), que mede a satisfação do indivíduo com o seu trabalho. O instrumento é unifatorial e contém cinco itens, a serem respondidos por meio de uma escala Likert de 5 pontos, variando de "discordo fortemente" (1) a "concordo fortemente" (5). Exemplo de item: sinto-me satisfeito com meu trabalho atual. No estudo de validação original, o instrumento obteve um índice de consistência interna (Alfa de Cronbach) de 0,80. No presente estudo, este índice foi de 0,93.

Para a coleta de informações sociodemográficas, foi utilizado um questionário com perguntas relativas aos seguintes aspectos: idade, sexo, escolaridade, profissão, estado civil e quantidade de filhos.

\section{Procedimentos}

Inicialmente, a pesquisa foi submetida ao Comitê de Ética em Pesquisa da instituição dos autores. Depois da aprovação, a coleta de dados foi realizada em versão impressa $(12,5 \%)$ e em versão on-line $(87,5 \%)$. Na versão impressa, os questionários foram entregues aos respondentes e preenchidos de forma individual ou coletiva, em seus locais de trabalho ou residência, sendo devolvidos em seguida. Tal aplicação durou em média 20 minutos. Na versão on-line, um formulário foi criado no aplicativo Google Docs, sendo os indivíduos convidados a participar por meio de mensagens no Facebook e no Linkedln. Em ambos os casos, os respondentes inicialmente manifestaram sua concordância em participar da pesquisa, mediante o preenchimento do Termo de Consentimento Livre e Esclarecido, e, em seguida, iniciaram a realização do questionário.

\section{Resultados}

Os testes $t$ de Student para amostras independentes, realizados com o intuito de verificar as diferenças porventura existentes entre as médias das cinco escalas compreendidas pela investigação, entre o grupo de participantes que responderam aos questionários na versão impressa e on-line, revelaram que não houve diferenças significativas entre as médias dos dois grupos em nenhuma das escalas.

A Tabela 1 apresenta as correlações entre os 18 itens da Escala de Enriquecimento Trabalho-Família. A fim de se verificar a estrutura da Escala de Enriquecimento TrabaIho-Família, procedeu-se à Análise Fatorial Confirmatória, por meio da Modelagem de Equações Estruturais. Baseando-se no fato de os diferentes estudos de validação do referido instrumento terem encontrado modelos de duas e de seis dimensões (Carlson et al., 2006; Hennessy, 2007; Lim, Choi, \& Song, 2012), foram inicialmente testados os modelos de uma, duas e seis dimensões independentes, com os seguintes índices de ajuste sendo analisados (Byrne, 2001): Qui-Quadrado (testa a probabilidade do modelo teórico se ajustar aos dados, e quanto maior o valor do $\chi 2$, pior o ajustamento); Root-Mean-Square Error of Approximation (RMSEA - deve se situar abaixo de 0,08, aceitando-se valores até 0,10$)$; Tucker-Lewis Index (TLI - são considerados aceitáveis 
valores acima de 0,90); Akaike Information Criterion (AIC - recomenda-se escolher o modelo de menor valor); Bayer Information Criterion (BIC - recomenda-se escolher o modelo de menor valor).

A análise dos resultados obtidos evidenciou, no entanto, que os índices de ajuste dos modelos de uma, de duas e de seis dimensões independentes não se mostraram aceitáveis (Tabela 2). Considerando-se que alguns itens eram muito semelhantes e, consequentemente, apresentaram-se altamente correlacionados (multicolinearidade), conforme pode ser observado na Tabela 1, optou-se pela exclusão daqueles $(1,3,5,7$, $10,11,14$ e 16) que apresentavam as maiores correlações.

\section{Tabela I. Correlação entre os itens da Escala de Enriquecimento Trabalho-Família}

\begin{tabular}{cccccccccccccccccc}
\hline Itens & 1 & 2 & 3 & 4 & 5 & 6 & 7 & 8 & 9 & 10 & 11 & 12 & 13 & 14 & 15 & 16 & 17 \\
\hline 2 & 0,82 & - & - & - & - & - & - & - & - & - & - & - & - & - & - & - & - \\
\hline 3 & 0,83 & 0,87 & - & - & - & - & - & - & - & - & - & - & - & - & - & - & - \\
\hline 4 & 0,62 & 0,66 & 0,63 & - & - & - & - & - & - & - & - & - & - & - & - & - & - \\
\hline 5 & 0,63 & 0,68 & 0,63 & 0,85 & - & - & - & - & - & - & - & - & - & - & - & - & - \\
\hline 6 & 0,68 & 0,65 & 0,65 & 0,83 & 0,84 & - & - & - & - & - & - & - & - & - & - & - & - \\
\hline 7 & 0,55 & 0,59 & 0,58 & 0,61 & 0,71 & 0,74 & - & - & - & - & - & - & - & - & - & - & - \\
\hline 8 & 0,53 & 0,61 & 0,59 & 0,67 & 0,78 & 0,72 & 0,88 & - & - & - & - & - & - & - & - & - & - \\
\hline 9 & 0,56 & 0,53 & 0,58 & 0,54 & 0,56 & 0,65 & 0,76 & 0,71 & - & - & - & - & - & - & - & - & - \\
\hline 10 & 0,59 & 0,56 & 0,59 & 0,55 & 0,54 & 0,58 & 0,49 & 0,49 & 0,54 & - & - & - & - & - & - & - & - \\
\hline 11 & 0,64 & 0,53 & 0,61 & 0,54 & 0,54 & 0,60 & 0,58 & 0,52 & 0,66 & 0,78 & - & - & - & - & - & - & - \\
\hline 12 & 0,59 & 0,64 & 0,65 & 0,60 & 0,59 & 0,60 & 0,53 & 0,57 & 0,56 & 0,77 & 0,75 & - & - & - & - & - & - \\
\hline 13 & 0,61 & 0,49 & 0,56 & 0,56 & 0,57 & 0,60 & 0,58 & 0,53 & 0,51 & 0,64 & 0,71 & 0,65 & - & - & - & - & - \\
\hline 14 & 0,58 & 0,52 & 0,58 & 0,57 & 0,58 & 0,65 & 0,60 & 0,61 & 0,59 & 0,69 & 0,69 & 0,75 & 0,84 & - & - & - & - \\
\hline 15 & 0,54 & 0,52 & 0,53 & 0,54 & 0,51 & 0,53 & 0,49 & 0,51 & 0,55 & 0,63 & 0,61 & 0,69 & 0,54 & 0,63 & - & - & - \\
\hline 16 & 0,58 & 0,54 & 0,58 & 0,57 & 0,60 & 0,60 & 0,61 & 0,66 & 0,62 & 0,60 & 0,65 & 0,69 & 0,63 & 0,68 & 0,67 & - & - \\
\hline 17 & 0,54 & 0,52 & 0,53 & 0,61 & 0,62 & 0,62 & 0,56 & 0,61 & 0,61 & 0,62 & 0,70 & 0,70 & 0,60 & 0,65 & 0,68 & 0,82 & - \\
\hline 18 & 0,56 & 0,52 & 0,54 & 0,60 & 0,55 & 0,59 & 0,55 & 0,57 & 0,55 & 0,60 & 0,64 & 0,69 & 0,69 & 0,72 & 0,56 & 0,75 & 0,75 \\
\hline
\end{tabular}

Fonte: Elaborada pelos autores.

Depois da exclusão desses itens, foram novamente testados os modelos de dois e seis fatores (independentes, correlacionados e bifactor), em razão de eles estarem associados à estrutura original da escala (dois fatores, com três dimensões cada). A análise desses resultados levou à opção pelo modelo de dois fatores na estrutura bifactor, 
em razão de seus melhores índices de ajuste (Tabela 2). Nesse modelo, cada um dos dois fatores (enriquecimento do trabalho para a família e enriquecimento da família para o trabalho) explicou parte da variância de um conjunto de itens a ele associado, enquanto um fator geral (enriquecimento trabalho-família) explicou a parte da variância dos itens não explicada pelos fatores (Reise, Moore, \& Haviland, 2010). A Figura 1 traz a representação gráfica do modelo retido, com suas cargas fatoriais padronizadas.

Tabela 2. Índices de ajuste de diferentes modelos para a Escala de Enriquecimento Trabalho-Família

\begin{tabular}{llllll}
\hline Fatores/Índices & TLI & RMSEA & AIC & BIC & $\begin{array}{l}\text { Qui-quadrado } \\
\text { (grau de liberdade) }\end{array}$ \\
\hline Um & 0,67 & 0,21 & 2641,58 & 2786,68 & $2569,58(135)$ \\
\hline Dois & 0,75 & 0,18 & 1993,66 & 2142,79 & $1919,66(134)$ \\
\hline Seis & 0,84 & 0,14 & 1320,65 & 1489,94 & $1236,65(129)$ \\
\hline Bifactor: dois & 0,93 & 0,10 & 212,23 & 333,15 & $152,2(25)$ \\
\hline Bifactor: seis & 0,86 & 0,14 & 1116,38 & 1334,04 & $1008,38(117)$ \\
\hline
\end{tabular}

Fonte: Elaborada pelos autores.

Figura I. Modelo Final da Escala de Enriquecimento Trabalho-Família, com as cargas fatoriais padronizadas

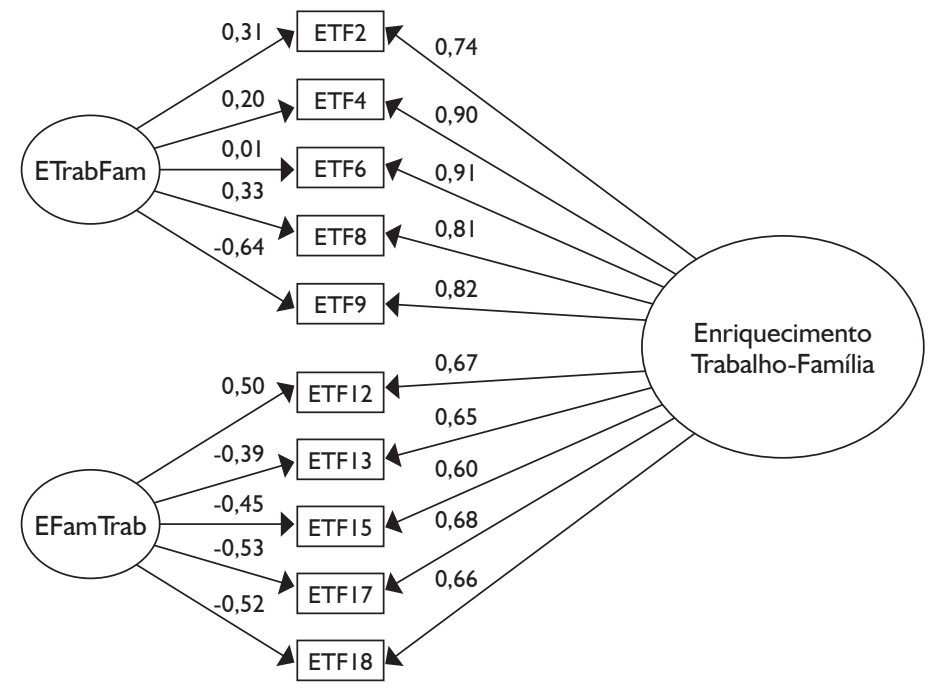

Fonte: Elaborada pelos autores. 
Em síntese, a versão brasileira da Escala de Enriquecimento Trabalho-Família ficou composta por dois fatores (Enriquecimento do Trabalho para a Família e Enriquecimento da Família para o Trabalho), com cinco itens cada. Os índices de consistência interna de ambos os fatores da escala, calculados por meio do Alfa de Cronbach, foram iguais a 0,90. O fator geral, por sua vez, apresentou um Alfa de Cronbach igual a 0,94.

Buscou-se, ainda, avaliar as evidências de validade de construto convergente e divergente da Escala de Enriquecimento Trabalho-Família. Para tanto, os escores obtidos em cada um dos instrumentos foram inicialmente computados e suas médias e desvios padrão, calculados (Tabela 3). Em seguida, foram investigadas as correlações ( $r$ de Pearson) dos escores da Escala de Enriquecimento Trabalho-Família com os escores globais das medidas de satisfação no trabalho e satisfação com a vida (validade convergente), bem como com a medida de conflito trabalho-família (validade divergente). $\mathrm{Na}$ análise dos coeficientes de correlação foram adotados os critérios de Miles e Shevlin (2001), que classificam a magnitude das correlações de acordo com os seguintes intervalos: 0,10-0,29 (baixa); 0,30-0,49 (moderada) e maior que 0,50 (elevada).

Os dados obtidos (Tabela 3 ) demonstraram que a subescala de Enriquecimento do Trabalho para a Família apresentou uma correlação positiva elevada $(r=0,73 ; p<0,01)$ com a Escala de Satisfação no Trabalho, o que confirma a Hipótese 1 e evidencia que quanto mais as pessoas percebem que o trabalho que desenvolvem beneficia a qualidade de sua vida em família, mais elas se sentem satisfeitas com seu trabalho. Observou-se, também, que a subescala de Enriquecimento da Família para o Trabalho apresentou uma correlação positiva elevada com a Escala de Satisfação com a Vida $(r=0,55 ; p<0,01)$, o que é coerente com a Hipótese 2 e sinaliza que quando a vida em família leva os indivíduos a auferirem maior qualidade de vida em seu trabalho, mais satisfeitas elas se sentem com a sua vida em geral.

Verificou-se, ainda, que a subescala de Enriquecimento Trabalho-Família apresentou uma correlação moderada negativa com a subescala de Interferência do Trabalho na Família $(r=-0,31 ; p<0,01)$, e que a subescala de Enriquecimento Família-Trabalho também demonstrou uma correlação moderada negativa com a subescala de Interferência da Família para o Trabalho $(r=-0,35 ; p<0,01)$. Tais dados impedem a confirmação das Hipóteses 3 e 4 e apontam para o fato de que os processos de enriquecimento e de conflito trabalho-família não se constituem em fenômenos totalmente distintos entre si.

\section{Tabela 3. Médias, desvios padrão e coeficientes de correlação entre as escalas do estudo}

\begin{tabular}{llllllll}
\hline Variáveis & Médias & $\begin{array}{c}\text { Desvios } \\
\text { Padrão }\end{array}$ & I & 2 & 3 & 4 & 5 \\
\hline $\begin{array}{l}\text { I. Enriquecimento do Trabalho para a } \\
\text { Família }\end{array}$ & 3,66 & 1,00 & - & - & - & - & - \\
\hline
\end{tabular}




\section{Tabela 3. Médias, desvio padrão e coeficientes de correlação entre as escalas do estudo (conclusão)}

\begin{tabular}{llllllll} 
Variáveis & Médias & $\begin{array}{c}\text { Desvios } \\
\text { Padrão }\end{array}$ & I & 2 & 3 & 4 & 5 \\
\hline $\begin{array}{l}\text { 2. Enriquecimento da Família para o } \\
\text { Trabalho }\end{array}$ & 3,83 & 0,92 & 0,78 & - & - & - & - \\
\hline 3. Interferência do Trabalho na Família & 3,00 & 1,53 & $-0,31$ & $-0,30$ & - & - & - \\
\hline 4. Interferência da Família no Trabalho & 2,57 & 1,24 & $-0,32$ & $-0,35$ & 0,59 & - & - \\
\hline 5. Satisfação com o Trabalho & 3,28 & 1,44 & 0,73 & 0,63 & $-0,32$ & $-0,30$ & - \\
\hline 6. Satisfação com a Vida & 4,80 & 1,16 & 0,59 & 0,55 & $-0,37$ & $-0,25$ & 0,64 \\
\hline
\end{tabular}

Fonte: Elaborada pelos autores.

\section{Discussão}

O presente estudo teve como objetivo investigar as evidências de validade de estrutura interna, convergente e divergente da Escala de Enriquecimento Trabalho-Família, bem como indicadores de sua consistência interna. No que diz respeito à análise fatorial confirmatória, os dados obtidos revelaram que o modelo de melhor ajuste foi o de estrutura bifactor, com uma dimensão geral associada ao enriquecimento trabalho-família, e dois fatores específicos, que agregam itens sobre o enriquecimento do trabalho para a família e o enriquecimento da família para o trabalho.

Tais resultados contrariam a pesquisa de Carlson et al. (2006), ao construírem o instrumento original, bem como as de Lim et al. (2012) e de Nicklin e McNall (2013). Assim é que esses autores verificaram ser o modelo de dois fatores, cada um com três dimensões, o que fornecia os melhores índices de ajuste e, no estudo atual, não foi possível reproduzir as três dimensões originais em cada fator.

Os resultados ora obtidos mostram-se, no entanto, até certo ponto, consistentes com o estudo de Hennessy (2007), que também obteve uma estrutura de dois fatores, embora não associados a um fator mais geral. Os dados obtidos por Hennessy (2007) levaram, inclusive, à exclusão de quatro itens em cada fator (escala de enriquecimento trabalho-família - itens 2, 6, 7, 8; escala de enriquecimento família-trabalho - itens $11,14,16,17)$, e alguns desses itens foram os também eliminados na presente investigação (escala de enriquecimento trabalho-família - itens 1, 3, 5, 7; escala de enriquecimento família-trabalho - itens 10, 11,14,16).

A estrutura apresentada na presente pesquisa representa, portanto, um avanço em relação à literatura na área de enriquecimento trabalho-família, ao propor um modelo mais complexo para a compreensão do referido construto. Em outras palavras, ainda que o enriquecimento ocorra no ambiente do trabalho e da família, a dimensão geral indica que o construto também abarca uma estrutura de enriquecimento geral. 
Tal modelo de dois fatores distintos mostra-se ainda congruente com a teoria de Greenhaus e Powell (2006), segundo a qual o fenômeno do enriquecimento trabalho-família é de natureza bidirecional, na medida em que as experiências adquiridas no trabalho podem promover a qualidade de vida na família e vice-versa.

$\mathrm{Na}$ investigação atual, o índice de consistência de ambos os fatores, calculados pelo Alfa de Cronbach, foi igual a 0,90. Esses resultados são semelhantes aos observados por Carlson et al. (2006), nas subescalas de enriquecimento do trabalho para a família $(\alpha=0,92)$ e de enriquecimento da família para o trabalho $(\alpha=0,86)$, bem como por Hennessy (2007), na subescala de enriquecimento do trabalho para a família $(\alpha=0,91)$. Na subescala de enriquecimento da família para o trabalho, porém, o autor (Hennessy, 2007) obteve um índice de consistência interna $(\alpha=0,79)$ inferior aos presentemente obtidos. Em relação ao fator geral, o Alfa de Cronbach encontrado na presente pesquisa $(\alpha=0,94)$ foi semelhante ao encontrado por Carlson et al. (2006) $(\alpha=0,92)$.

No que tange à validade convergente (nomological validity) das duas dimensões da escala, observou-se uma correlação positiva elevada entre o enriquecimento do trabaIho para a família e a satisfação com o trabalho, bem como entre o enriquecimento da família para o trabalho e a satisfação com a vida, o que confirmou as Hipóteses 1 e 2. Tais resultados mostram-se consistentes com achados dos diferentes estudos (Hennessy, 2007; McNall, Nicklin, \& Song, 2010; Nicklin \& McNall, 2013) que também constataram associações positivas entre esses construtos, apesar de nessas investigações o enriquecimento da família para o trabalho ter sido correlacionado com a satisfação com a família e não com a satisfação com a vida.

As correlações altas e positivas observadas entre o enriquecimento do trabalho para a família e a satisfação com o trabalho e entre o enriquecimento da família para o trabalho e a satisfação com a vida encontram apoio teórico no argumento de que para o enriquecimento ocorrer, o indivíduo deve adquirir recursos em um determinado domínio, o que, por sua vez, leva ao aumento da satisfação nesse domínio (Hennessy, 2007; McNall, Nicklin, \& Masuda, 2010; Nicklin \& McNall, 2013). Dessa forma, um indivíduo que experimenta enriquecimento do trabalho para a família tende a ficar mais satisfeito em seu trabalho, porque ele o está provendo de recursos. De modo semelhante, se o indivíduo experimentar o enriquecimento da família para o trabalho ficará mais satisfeito com sua família, por essa lhe prover os recursos. Em relação à validade divergente dos fatores do instrumento, foram encontradas correlações moderadas e negativas entre o enriquecimento do trabalho para a família e a interferência do trabalho na família, e entre o enriquecimento da família para o trabalho e a interferência da família no trabalho, o que impediu a confirmação das Hipóteses 3 e 4 , que previam correlações baixas entre os referidos construtos. Tais dados divergem dos anteriormente obtidos por Hennessy (2007), que observou a existência de uma correlação baixa e negativa entre os dois construtos mencionados $(r=-0,21)$. Além disso, eles permitem concluir que o enriquecimento trabalho-família e o conflito trabalho-família possuem variância compartilhada, embora se caracterizem como di- 
ferentes consequências dos processos de gerenciamento dos domínios do trabalho e da família (Hennessy, 2007). Tornam-se, assim, necessários estudos futuros que possam avaliar se esse foi um resultado específico à presente amostra ou se constitui em uma característica da realidade brasileira.

Em síntese, a escala de Enriquecimento Trabalho-Família apresentou evidências de validade de estrutura, convergente e divergente, bem como consistência interna, adequadas, em uma amostra brasileira. O referido instrumento pode ser assim considerado como uma medida apropriada à avaliação de como os recursos adquiridos no trabalho melhoram o desempenho na família e vice-versa, o que recomenda seu uso para fins de pesquisas.

Cumpre registrar, porém, uma limitação do estudo. Assim é que o fato de a amostra ter sido composta por participantes em sua maioria do estado do Rio de Janeiro dificulta a generalização dos resultados para os demais estados brasileiros. No que tange à realização de estudos futuros, sugere-se que sejam realizadas pesquisas que objetivem o aprofundamento da rede nomológica do construto enriquecimento trabalho-família, em especial, no que diz respeito a seu impacto em variáveis que caracterizam o bem-estar e a qualidade de vida no trabalho e fora dele, como os afetos dirigidos ao trabaIho, o engajamento no trabalho, a satisfação com a família etc. Tais pesquisas poderiam ser de natureza longitudinal, o que permitiria maior compreensão das relações entre essas variáveis.

\section{Referências}

Aguiar, C. V. N., \& Bastos, A. V. B. (2013). Tradução, adaptação e evidências de validade para a medida de conflito trabalho-família. Avaliação Psicológica, 12(2), 203-212.

Borsa, J. C., Damasio, B. F., \& Bandeira, D. R. (2012). Adaptação e validação de instrumentos psicológicos entre culturas: algumas considerações. Paidéia, 22(53), 423-432.

Byrne, B. M. (2001). Structural equation modeling with AMOS: Basic concepts, applications, and programing. New Jersey: Lawrence Erlbaum Associates.

Carlson, D. S., Kacmar, M., Wayne, J. H., \& Grzywacz, J. G. (2006). Measuring the positive side the work-family interface: development and validation of a work-family enrichment scale. Journal of Vocational Behavior, 68(1), 131-164.

Diener, E., Emmons, R. A., Larsen, R. J., \& Griffin, S. (1985). The satisfaction with life scale. Journal of Personality Assessment, 49(1), 71-75.

Emmons, R. A. (1986). Personal strivings: An approach to personality and subjective well-being. Journal of Personality and Social Psychology, 51(5), 1058-1068.

Gouveia, V. V., Chaves, S. S. S., Oliveira, I. C. P., Dias, M. R., Gouveia, R. S. V., \& Andrade, P. R. (2003). A utilização do QSG-12 na população geral: estudo de sua validade de construto. Psicologia: Teoria e Pesquisa, 19(3), 241-238. 
Greenhaus, J. H., \& Parasuraman, S. (1999). Research on work, family, and gender: current status and future directions. In G. N. Powell (Ed.), Handbook of gender and work (pp. 291-412). Newbury Park: Sage.

Greenhaus, J. H., \& Powell, G. N. (2006). When work and family are allies: a theory of work- family enrichment. Academy of Management Review, 31(1), 72-92.

Grzywacz, J. G. (2002). Work-family spillover and health during midlife: is managing conflict everything? American Journal of Health Promotion, 14(4), 236-243.

Hennessy, K. D. (2007). Work-family balance: An exploration of conflict and enrichment for women in a traditional occupation. Tese de doutorado, University of Maryland, Maryland, Estados Unidos.

Lim, D. H., Choi, M., \& Song, J. H. (2012). Work-family enrichment in Korea: construct validation and status. Leadership \& Organization Development Journal, 33(3), 282-299.

McNall, L. A., Nicklin, J. M., \& Masuda, A. (2010). A meta-analytic review of the consequences associated with work family enrichment. Journal of Business and Psychology, 25(3), 381-396.

Miles, J. N. V., \& Shevlin, M. E. (2001). Applying regression and correlation: a guide for students and researchers. London: Sage Publications.

Netemeyer, R. G., Boles, J. S., \& McMurrian, R. (1996). Development and validation of work-family conflict and family-work conflict scales. Journal of Applied Psychology, 81(4), 400-410.

Nicklin, J. M., \& McNall, L. A. (2013). Work-family enrichment, support, and satisfaction: A test of mediation. European Journal of Work and Organizational Psychology, 22(1), 67-77.

Poelmans, S., Stepanova, E., \& Masuda, A. (2008). Spillover between personal and professional life: Definitions, antecedents, consequences, and strategies. In $\mathrm{K}$. Korabic, D. Lero \& D. Whitehead (Ed.). Handbook of work and family (pp. 141-156). London: Elsevier.

Reise, S. P., Moore, T. M., \& Haviland, M. G. (2010). Bifactor models and rotations: Exploring the extent to which multidimensional data yield univocal scale scores. Journal of Personality Assessment, 92(6), 544-559.

Silva, A. P. C., \& Ferreira, M. C. (2009, julho). Escala de Satisfação Geral no Trabalho. Anais do Congresso Brasileiro de Avaliação Psicológica, Campinas, SP, Brasil, 4.

Weiss, H. M. (2002). Deconstructing job satisfaction: separating evaluations, beliefs and affective experiences. Human Resource Management Review, 12(2), 173-194. 